\title{
Effects of Recent Carrot-and-Stick Policy Initiatives on Private Health Insurance Coverage in Australia
}

\author{
Alfons Palangkaraya and Jongsay Yong \\ Melbourne Institute of Applied Economic and Social Research \\ and \\ Centre for Microeconometrics \\ The University of Melbourne
}

Melbourne Institute Working Paper No. 20/04

ISSN 1328-4991 (Print)

ISSN 1447-5863 (Online)

ISBN 0734031629

August 2004

Melbourne Institute of Applied Economic and Social Research

The University of Melbourne

Victoria 3010 Australia

Telephone (03) 83442100

Fax (03) 83442111

Email melb-inst@unimelb.edu.au

WWW Address http://www.melbourneinstitute.com 


\begin{abstract}
The Australian government implemented a sequence of new policy initiatives during 1997-2000 with a stated aim of raising the take-up rate of private health insurance (PHI). Taken together, it is quite clear that these new policy initiatives were effective. The overall proportion of Australian population with private health insurance cover increased by more than $35 \%$. However, much less clear is the effectiveness of different components of the policies. This lacks of clarity results from the sequential implementation those policies. Because of the potentially large cost differences in their implementation, a better understanding of the each policy's effects can be valuable for future policymaking. This paper attempts to isolate the effects of those different policies using the 1995 and 2001 National Health Survey data. These two datasets allow the estimation of private health insurance demands before and after the policy changes. Consequently, they also allow for a counterfactual analysis of what would have happened had there been no new policies. Combined with the age-specific aspect Lifetime Health Cover (LHC), the counterfactual analysis indicates that the effects of LHC fall between $42 \%$ and $75 \%$ of the overall increase in PHI membership.
\end{abstract}




\section{Introduction}

The Australian health care system is based on an universal access principle, under which every person regardless of income is entitled to be a member of Medicare, a universal health insurance scheme. However, private health insurance (PHI) has always been a prominent feature of the Australian health system, despite the availability of a publicly funded alternative since $1975 .{ }^{1}$ For much of the 1990 s, some $30 \%$ to $40 \%$ of the population is covered by PHI. It is, however, plainly obvious that the coverage of PHI has been on a declining trend since the introduction of Medibank in 1975. There are a variety of reasons, not least of which is the appeal of the publicly funded alternative (Medibank/Medicare) to the masses.

With a purported goal of reducing the burden on public hospitals, the Australian government implemented a sequence of new policies during 1997-2000. The three major policy initiatives are, in chronological order: (i) The Private Health Insurance Incentives Scheme (PHIIS), introduced in 1997, which imposes a tax levy on high-income earners who do not have PHI, and a means-tested subsidy scheme for low-income earners who purchase PHI. (ii) A 30\% premium rebate, introduced in 1999, to replace the means-tested subsidy under PHIIS. The $30 \%$ rebate is non-means tested, and applies to all private health insurance policies, including existing ones that were already in place. (iii) Lifetime Health Cover (LHC), introduced in 2000, permits a limited form of agerelated risk rating by private health insurance funds. Under LHC, insurance funds are allowed to discriminate consumers by age at time of entry. The immediate aim of these policies was to raise the take-up rate of private health insurance (PHI). As a result of these policies, the proportion of the population with private hospital cover increased from $31 \%$ in 1999 to more than $45 \%$ in 2001, an increase of more than 14 percentage points in two years. Two components of the new policies, the $30 \%$ premium rebate and Lifetime Health Cover (LHC), have been widely regarded as the most effective in raising the PHI take-up rate.

\footnotetext{
${ }^{1}$ The first universal health insurance system was announced in 1972 and put in place in 1975 under the name of Medibank. The name was changed to Medicare in 1984 following some major revamps of the scheme.
} 
It is not clear, however, what proportion of the increase can be attributed to which of those policies. Butler (2002) examines the trend in the proportion of population with private hospital insurance cover between June 1984 and March 2002. By noting the timing of the sequential policies introduced beginning in mid-1997, he argues that it was LHC that induced the bulk of the increase in PHI take-up rate. Using a similar policy timing idea but with a more rigorous trend analysis, Frech et al. (2003) attempt to measure the relative impact of the different policies. They estimate that the $30 \%$ rebate lead to an $11 \%$ increase in PHI demand. However, they were not able to disentangle the effects of the rebate and the LHC.

This difficulty in discerning the separate effects of the policies can have significant consequences when the cost differential across policies is large. In fact, the substantial cost differences between the two policies have attracted heated debate among academics and policymakers as to the cost-effectiveness of each policy. Duckett and Jackson (2000), for example, estimate that the $30 \%$ rebate costs more than $\$ 2$ billions per year while LHC costs practically nothing to the government. Therefore, from a cost efficiency point of view, it is clearly desirable to be able to distinguish the contributions of the two policies for a more cost effective policymaking.

This paper departs from previous studies by using micro-level cross-section data to disentangle the effects of the two policies. We argue that although a significant proportion of the increase in PHI coverage occurred after the introduction of LHC, this policy was introduced on top of, not in place of, the $30 \%$ premium rebate and the tax levy under PHIIS. This means that the separate contributions of these policies cannot be readily inferred from time series data. Using cross-sectional micro-level data, this paper contributes to the discussion by providing separate estimates of the effects of the $30 \%$ rebate and LHC. As a significant improvement over previous studies, this paper estimates micro-level model of PHI demand and takes advantage of the design of the two policies to separate their effects.

Using unit record data from the 1995 and 2001 National Health Survey (NHS) data, in this paper we estimate PHI demand equations before and after the implementation 
of the new policies separately for single individuals and families. This allows us to construct a counterfactual scenario to measure the effects of the new policies on PHI take-up rate for those people who are highly unlikely to be covered without these new policies. Furthermore, utilizing the age criterion of the LHC policy, we are able to isolate the contribution of LHC. For singles, we find that the LHC accounts for at least $42 \%$ and at most $61 \%$ of the total increase in take-up rate. For families, the corresponding figures are $42 \%$ and $78 \%$ of the total increase, respectively. The total effects of LHC, inclusive of singles and familes, are estimated to be between $42 \%$ and $75 \%$ of the total increase. Therefore, while these figures show the significant impact of LHC on PHI take-up, they are much lower than those suggested by previous studies.

The rest of this paper proceeds as follows. In section 2, we provide a brief discussion on the changes in the private health insurance policies. This will be followed by the empirical model specifications and an explanation of data and variable construction in section 3. In section 4 we discuss and interpret the empirical results. Section 5 concludes the paper.

\section{PHI Policy Changes}

There are three major sets of PHI policy changes implemented between 1997 and 2000 which may affect PHI demand. The first set, introduced in 1997, includes a meanstested PHI premium subsidy and a 1\% Medicare tax surcharge for high income earners who is not covered by an approved PHI policy. Then, in 1999, the means tested rebate was replaced with a non-means tested $30 \%$ premium rebate for all PHI policies. Finally, introduced in 2000, are Lifetime Health Cover (LHC), which allow health funds to discriminate consumers according to their age of entry into the fund. Because of the continuing decline in PHI take-up rate even after the introduction of the first set of policies, the $30 \%$ premium rebate and particularly LHC, have been widely regarded as the most effective policies and therefore are the focus of most earlier studies.

The basic idea of the LHC scheme is to induce the low risk population back into the 
private health insurance system. It was mainly a response to the findings that more and more low-risk individuals were leaving the PHI system, resulting in a system consisting mostly of the high-risk groups-a phenomenon often referred to as the "adverse selection spiral" (see for example, Industry Commission, 1997). Consequently, the scheme was designed around a financial penalty for the low risk groups. The target group was individuals between 30 and 65 years old. The amount of the penalty is set at $2 \%$ of the premium for each year beyond the age of 30 for anyone in the targeted population entering a health fund for the first time.

As can be seen from Figure 1, immediately following the introduction of LHC, PHI membership jumped from $31 \%$ of the population at the end of 1999 to more than $45 \%$ at the end of 2000. Thus, one is naturally tempted to attribute most of the effects to LHC. However, there are two plausible reasons why the effects of the $30 \%$ rebate should not be discounted entirely. First, only approximately $12 \%$ of the 2001 NHS respondents regarded LHC as one of the reasons for having PHI (see Figure 2-a). Compared to Medicare Levy, for example, LHC is clearly not a significantly more important reason for having PHI. Second, LHC has a very specific target group which accounts for less than $72 \%$ of private health insurance membership in 2001. More importantly, a significant proportion of the target groups were in fact already covered by private health insurance before those policy initiatives were implemented. For example, approximately $60 \%$ of single individuals in 2001 who have PHI cover have had it for more than 5 years period. Finally, consumer theory asserts that what matters in the end is whether or not having PHI is optimal given an individual's budget constraint. In fact, slightly more than $60 \%$ of families without PHI cited costs as a reason why they did not purchase PHI (see Figure 2-b). It is then quite plausible that the jump following the introduction of LHC would have been much smaller if PHI were still unaffordable in the absence of the rebate.

\section{Empirical Framework and Data}

The specification of our empirical models follows Cameron and Trivedi (1991), and is quite similar to other earlier Australian based studies such as Hopkins and Kidd 
Figure 1: Proportion of Population with Private Health Insurance, 1996-2003

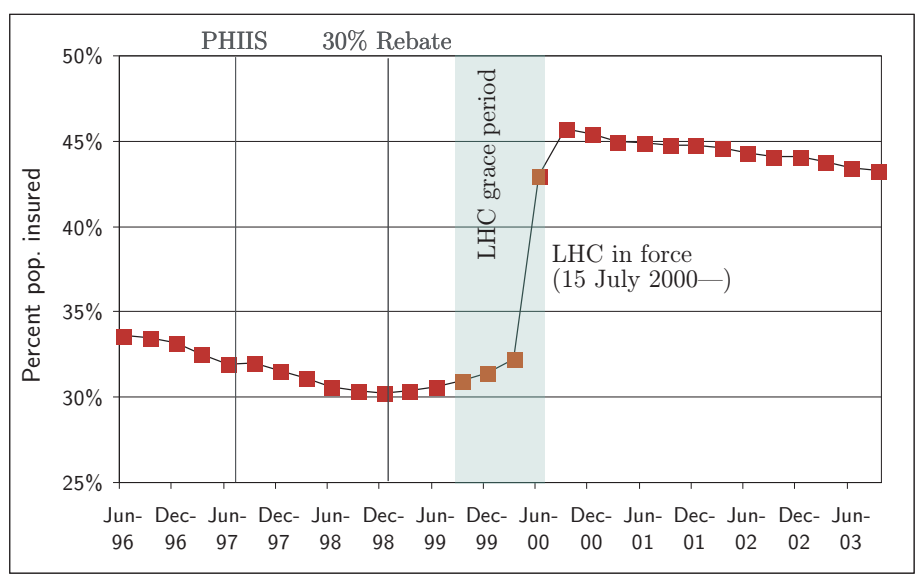

Figure 2: Reasons for having (a) and not having (b) Private Health Insurance, 2001

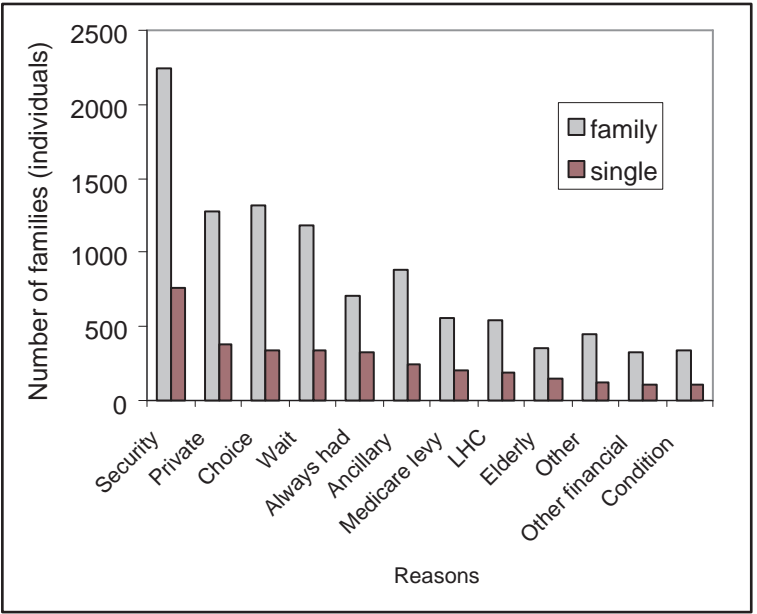

(a)

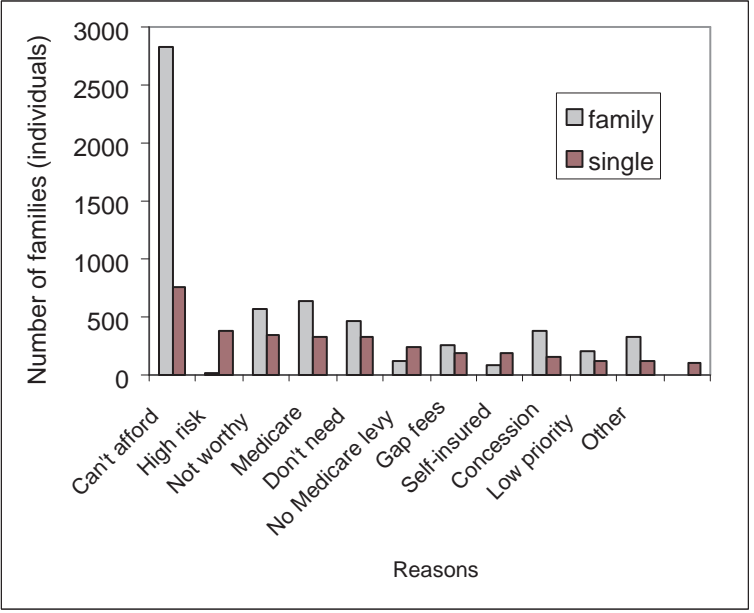

(b) 
(1996) and Barret and Conlon (2003). The basic framework is motivated by the theory of consumer choice under uncertainty, where an individual only purchases PHI if the expected utility from purchasing exceeds that from not purchasing. The decision to purchase PHI can thus be specified as a discrete choice model. That is, defining the binary variable PHI as an observed random variable with value 1 if PHI is chosen, and value 0 otherwise. The probability of purchasing PHI is

$$
\operatorname{Pr}[\mathrm{PHI}=1]=f(x, \beta)+e,
$$

where $x$ is a vector of observable individual characteristics, $\beta$ is the corresponding vector of parameters to be estimated, and $e$ is a random term representing the unobservables. We specify $f$ so that (1) can be estimated as a standard logit model. Following the three studies cited above, $x$ contains variables that capture variations in (i) expected medical needs (gender, family size, age, doctor visits); and (ii) risk aversion, preference as well as other socio-economic background (smoking, income, types of employment, residential area, and education). Missing from the list is the rate of insurance premium, which unfortunately is not available from existing Australian data sets.

Separately for singles and families, we estimate two sets of empirical models based on equation (1) twice, one for 1995 and one for 2001. The 2001 estimates provides variations in the marginal effects and the predicted probabilities across age groups. The 1995 estimation provides us with the average decision rules used by individuals before the set of the policy changes were in place. Thus, by applying the estimated 1995 "decision rule" on the 2001 population, we obtain estimates of what would have happened if there were no policy changes between the two period. Based on these estimates, we decompose the observed proportion of population with PHI in 2001 into (i) those who would have purchased PHI even when there were no policy change, and (ii) those whose purchasing decision was more of a response to the new policies. Furthermore, utilizing the age dependent design of the LHC policy, we separate the impact of LHC from that of the $30 \%$ premium rebates. This is done by taking the difference in the average probabilities between the LHC target group (age 30-69) and the non-target group (age 18-29). ${ }^{2}$

\footnotetext{
${ }^{2}$ The actual target group is 30-65. The consideration of a wider band of the target group is to avoid the possibility of underestimating LHC effects due to any confusion on policy applicability for people of
} 
Table 1: List of variables in logit estimation

\section{Dependent variable}

PHI Private health insurance $(1=$ Yes, $0=$ No $)$.

$1=$ if hospital cover (with or without ancillary) is purchased.

(for family, $1=$ if at least one family member has hospital cover).

\section{Explanatory variables}

female dummy, $1=$ female (not applicable for family units).

immig dummy, $1=$ immigrant.

goverd dummy, 1=government concession/entitlement card holder.

stdinc standardised value of annualised weekly income (actual - mean income).

stdinc2 squared standardised income.

famsize number of persons living in the household (not applicable for singles).

childlt6 number of children of age $\leq 6$ years (not applicable for singles).

degree dummy, 1=has a Bachelor degree or higher.

diploma dummy, 1=has an associate diploma.

postscd dummy, 1=has basic, skilled, and/or other post-secondary qualifications.

admin dummy, $1=$ occupation is in an administrative position.

trade dummy, $1=$ occupation is trade.

clrksrv dummy, $1=$ occupation is clerical or services.

prof dummy, $1=$ occupation is in the professional category.

paraprof dummy, $1=$ occupation is paraprofessional.

plantop dummy, $1=$ occupation is categorized as plant operator.

drvisit1-4 dummy variables, $1=$ has a doctor visit within last 2 weeks,

2 weeks- -3 months, 3-6 months, 6-12 months.

chdrvis1-4 Defined similarly as above for the children (not applicable for singles).

chnum number of long-term chronic conditions.

chnumcld number of long-term chronic conditions of the children

(not applicable for singles).

smoker dummy, $1=$ current regular smoker. 
The data for the estimation come from the Confidentialised Unit Record Files of the 1995 and the 2001 National Health Surveys. The interview of the 2001 survey took place between February and November 2001. Therefore, we are confident that it captured most, if not all, of the effects of PHI policy changes. In their original formats, these data sets contain respectively 26,862 and 53,828 unit records for 2001 and 1995. Given the plausibly different PHI decisions between singles and families, we split the data files accordingly and estimate (1) separately for singles and families in each survey.

In addition, since the 2001 survey only randomly interviewed a single adult from a family type of households while the 1995 survey interviewed all adults in the sampled households, we randomly select a single adult from households in the 1995 data. This is to ensure that the two samples are comparable across time. Finally, there are several data cleaning steps that we take. These include dropping full-time students, non-family members, age below 18 for singles and age below 20 for families, and singles below age 22 who may be covered by family PHI. After all of these data preparation steps, we end up with useable records of, respectively for the 1995 and 2001 NHS, 4,648 and 4,710 singles and 7,059 and 9,543 families.

Table 1 lists the variables used in the estimation. Note that all person description variables in the case of family units refer to the characteristics of the selected adult of the particular family. In addition to variables listed in Table 1, we also include 13 regional dummy variables, which denote whether the unit's residence is in a metro or rural area in each state/territory, and 13 age dummy variables, with value 1 if the unit's age falls in the indicated age interval.

\section{Results}

Table 2 summarizes the estimated coefficients of the PHI demand model as specified in (1), for singles and families, and in 1995 and 2001. As can be seen from the table, most

age slightly older than 65. As will be argued later in the paper, even if this results in an overestimation of LHC effects, the estimated effects are still less than what commonly believed. 
of the usual determinants of PHI demand are significant and have the expected signs. Demand is increasing in income, but this effect is diminishing as suggested by the negative sign of the squared income term. This probably captures the preference for private health care and at the same time the ability to afford the costs of the insurance. Comparing the coefficients of 1995 and 2001, we see that the signs of estimated coefficients are mostly as expected and consistent in both years. For examples, the coefficients for gender, income, education, occupation, risk aversion, and health risks are quite stable in both years. There is no variable which shows a significant reversal in sign from positive significant to negative significant or vice versa.

Table 2: Coefficient Estimates of Logit Private Health Insurance Demand Models

\begin{tabular}{|c|c|c|c|c|c|c|c|c|}
\hline \multirow{3}{*}{$\begin{array}{l}\text { Variables } \\
\text { female }\end{array}$} & \multicolumn{4}{|c|}{ Single } & \multicolumn{4}{|c|}{ Family } \\
\hline & \multicolumn{2}{|c|}{1995} & \multicolumn{2}{|c|}{2001} & \multicolumn{2}{|c|}{1995} & \multicolumn{2}{|c|}{2001} \\
\hline & 0.2490 & $(0.0854)$ & 0.4677 & $(0.0803)$ & & & & \\
\hline famsize & & & & & 0.1650 & $(0.0430)$ & -0.0348 & $(0.0356)$ \\
\hline childlt6 & & & & & -0.1502 & $(0.0911)$ & 0.0905 & $(0.0750)$ \\
\hline nochild & & & & & 0.3293 & $(0.1012)$ & -0.1830 & $(0.0918)$ \\
\hline immig & -0.5881 & $(0.0992)$ & 1.0130 & $(0.8624)$ & -0.8946 & $(0.0646)$ & -0.4384 & $(0.3985)$ \\
\hline goverd & -1.1639 & $(0.1573)$ & -1.1936 & $(0.1338)$ & -1.3652 & $(0.0826)$ & -0.9957 & $(0.0780)$ \\
\hline stdinc & 0.7539 & $(0.0867)$ & 0.8457 & $(0.0814)$ & 0.1696 & $(0.0389)$ & 0.9441 & $(0.0495)$ \\
\hline stdinc2 & -0.1512 & $(0.0285)$ & -0.0791 & $(0.0217)$ & 0.1103 & $(0.0233)$ & -0.0831 & $(0.0256)$ \\
\hline vicmet & 0.2999 & $(0.1331)$ & 0.3749 & $(0.1311)$ & 0.0893 & $(0.1008)$ & 0.1117 & $(0.0928)$ \\
\hline qldmet & 0.1236 & $(0.1960)$ & 0.0825 & $(0.1649)$ & -0.1989 & $(0.1413)$ & -0.0411 & $(0.1072)$ \\
\hline samet & 0.7530 & $(0.1449)$ & 0.4565 & $(0.1431)$ & 0.2704 & $(0.1115)$ & 0.4103 & $(0.1113)$ \\
\hline wamet & 0.5212 & $(0.1733)$ & 0.6458 & $(0.1443)$ & 0.0869 & $(0.1274)$ & 0.4299 & $(0.1048)$ \\
\hline tasmet & -0.0334 & $(0.3384)$ & 0.6384 & $(0.2115)$ & 0.5051 & $(0.2341)$ & 0.8122 & $(0.1663)$ \\
\hline nt & 0.3271 & $(0.1789)$ & -0.3019 & $(0.2967)$ & 0.1023 & $(0.1331)$ & -0.0526 & $(0.2159)$ \\
\hline act & -0.1935 & $(0.1663)$ & 0.1792 & $(0.1619)$ & -0.2587 & $(0.1288)$ & -0.1387 & $(0.1115)$ \\
\hline nswrur & -0.1934 & $(0.2558)$ & -0.0283 & $(0.1612)$ & -0.3926 & $(0.1634)$ & -0.0394 & $(0.1072)$ \\
\hline vicrur & 0.1967 & $(0.2143)$ & 0.0436 & $(0.1852)$ & -0.4134 & $(0.1481)$ & -0.1003 & $(0.1182)$ \\
\hline & & & & & & & hued on $n$ & xt page... \\
\hline
\end{tabular}




\begin{tabular}{|c|c|c|c|c|c|c|c|c|}
\hline \multirow{3}{*}{$\begin{array}{l}\text { Variables } \\
\text { qldrur }\end{array}$} & \multicolumn{4}{|c|}{ Single } & \multicolumn{4}{|c|}{ Family } \\
\hline & \multicolumn{2}{|c|}{1995} & \multicolumn{2}{|c|}{2001} & \multicolumn{2}{|c|}{1995} & \multicolumn{2}{|c|}{2001} \\
\hline & 0.2227 & $(0.1787)$ & 0.0617 & $(0.1561)$ & -0.0222 & $(0.1298)$ & 0.2223 & $(0.1028)$ \\
\hline sarur & 0.0576 & $(0.2587)$ & -0.2687 & $(0.2298)$ & -0.4952 & $(0.1571)$ & 0.0251 & $(0.1541)$ \\
\hline warur & 0.4528 & $(0.3529)$ & 0.1089 & $(0.2548)$ & -0.1093 & $(0.1875)$ & 0.1678 & $(0.1528)$ \\
\hline tasrur & 0.5069 & $(0.2279)$ & -0.0892 & $(0.2152)$ & -0.2500 & $(0.1720)$ & 0.2487 & $(0.1385)$ \\
\hline age1819 & -0.7058 & $(0.2556)$ & -1.3256 & $(0.3916)$ & & & & \\
\hline age2024 & -0.5952 & $(0.1870)$ & -0.9805 & $(0.2070)$ & -0.8098 & $(0.1636)$ & -1.6430 & $(0.1747)$ \\
\hline age2529 & -0.7007 & $(0.1949)$ & -1.0732 & $(0.1883)$ & -0.6603 & $(0.1274)$ & -1.0879 & $(0.1155)$ \\
\hline age3034 & -0.4292 & $(0.2089)$ & -0.1289 & $(0.1846)$ & -0.3250 & $(0.1126)$ & -0.4373 & $(0.1024)$ \\
\hline age3539 & -0.0543 & $(0.2156)$ & 0.1032 & $(0.1927)$ & -0.1857 & $(0.1051)$ & -0.2344 & $(0.0927)$ \\
\hline age4549 & -0.0394 & $(0.2220)$ & 0.2628 & $(0.1882)$ & 0.3269 & $(0.1094)$ & 0.2634 & $(0.1006)$ \\
\hline age5054 & 0.4454 & $(0.2388)$ & 0.3608 & $(0.1839)$ & 0.5895 & $(0.1278)$ & 0.5293 & $(0.1162)$ \\
\hline age5559 & 0.7154 & $(0.2526)$ & 0.6268 & $(0.1963)$ & 1.2171 & $(0.1450)$ & 0.8211 & $(0.1321)$ \\
\hline age6064 & 1.3306 & $(0.2549)$ & 0.7926 & $(0.2020)$ & 1.2532 & $(0.1601)$ & 1.0693 & $(0.1385)$ \\
\hline age6569 & 1.4676 & $(0.2560)$ & 1.0976 & $(0.2110)$ & 1.5729 & $(0.1691)$ & 1.4716 & $(0.1501)$ \\
\hline age7074 & 1.5527 & $(0.2619)$ & 1.0492 & $(0.2065)$ & 1.6152 & $(0.1764)$ & 1.6224 & $(0.1538)$ \\
\hline age7579 & 1.6471 & $(0.2589)$ & 0.8378 & $(0.2120)$ & 1.4840 & $(0.2138)$ & 0.9422 & $(0.1691)$ \\
\hline age80p & 1.5411 & $(0.2594)$ & 1.0070 & $(0.2055)$ & 1.5241 & $(0.2747)$ & 1.0628 & $(0.2070)$ \\
\hline degree & 0.2789 & $(0.1372)$ & 0.4850 & $(0.1252)$ & 0.4173 & $(0.1021)$ & 0.5120 & $(0.0884)$ \\
\hline diploma & -0.0025 & $(0.1479)$ & 0.4031 & $(0.1344)$ & 0.2518 & $(0.0999)$ & 0.3701 & $(0.0873)$ \\
\hline postscd & 0.2375 & $(0.1003)$ & 0.2685 & $(0.0905)$ & 0.0848 & $(0.0700)$ & 0.1453 & $(0.0603)$ \\
\hline admin & 0.2777 & $(0.2132)$ & 0.7642 & $(0.2176)$ & 0.7530 & $(0.1181)$ & 0.6337 & $(0.1248)$ \\
\hline trade & 0.0109 & $(0.1702)$ & -0.1349 & $(0.1907)$ & 0.1020 & $(0.1112)$ & -0.0370 & $(0.1045)$ \\
\hline prof & 0.2484 & $(0.1867)$ & 0.2224 & $(0.1727)$ & 0.5331 & $(0.1197)$ & 0.3187 & $(0.1009)$ \\
\hline paraprof & 0.0467 & $(0.2259)$ & 0.3382 & $(0.1827)$ & 0.5854 & $(0.1386)$ & 0.5289 & $(0.1036)$ \\
\hline clrksrv & 0.3099 & $(0.1425)$ & 0.3032 & $(0.1412)$ & 0.5462 & $(0.0846)$ & 0.4175 & $(0.0770)$ \\
\hline plantop & -0.2266 & $(0.2478)$ & -0.1256 & $(0.2063)$ & 0.1721 & $(0.1451)$ & -0.3568 & $(0.1183)$ \\
\hline drvisit1 & 0.5043 & $(0.1390)$ & 0.2887 & $(0.1305)$ & 0.2329 & $(0.0968)$ & 0.1767 & $(0.0845)$ \\
\hline
\end{tabular}




\begin{tabular}{|c|c|c|c|c|c|c|c|c|}
\hline \multicolumn{9}{|c|}{ continued from previous page } \\
\hline \multirow[t]{2}{*}{ Variables } & \multicolumn{4}{|c|}{ Single } & \multicolumn{4}{|c|}{ Family } \\
\hline & \multicolumn{2}{|c|}{1995} & \multicolumn{2}{|c|}{2001} & \multicolumn{2}{|c|}{1995} & \multicolumn{2}{|c|}{2001} \\
\hline drvisit2 & 0.3347 & $(0.1304)$ & 0.2949 & $(0.1261)$ & 0.1585 & $(0.0896)$ & 0.1669 & $(0.0812)$ \\
\hline drvisit3 & 0.4567 & $(0.1448)$ & 0.2341 & $(0.1418)$ & 0.1374 & $(0.1013)$ & 0.2790 & $(0.0900)$ \\
\hline drvisit4 & 0.3406 & $(0.1537)$ & 0.2039 & $(0.1522)$ & 0.2009 & $(0.1070)$ & 0.2942 & $(0.0944)$ \\
\hline chnum & 0.0350 & $(0.0196)$ & 0.0703 & $(0.0259)$ & 0.0196 & $(0.0149)$ & 0.0533 & $(0.0172)$ \\
\hline chnumcld & & & & & 0.0061 & $(0.0460)$ & 0.0580 & $(0.0422)$ \\
\hline chdrvis1 & & & & & 0.4360 & $(0.1754)$ & 0.1548 & $(0.1555)$ \\
\hline chdrvis2 & & & & & 0.3993 & $(0.1637)$ & 0.0777 & $(0.1417)$ \\
\hline chdrvis3 & & & & & 0.3510 & $(0.1867)$ & 0.0123 & $(0.1550)$ \\
\hline chdrvis4 & & & & & 0.0618 & $(0.1994)$ & 0.0121 & $(0.1740)$ \\
\hline smoker & -0.6683 & $(0.0940)$ & -0.6513 & $(0.0885)$ & -0.5470 & $(0.0698)$ & -0.6587 & $(0.0614)$ \\
\hline constant & -1.5804 & $(0.2494)$ & -2.3054 & $(0.8895)$ & -1.1047 & $(0.2178)$ & 0.3206 & $(0.4356)$ \\
\hline $\mathrm{N}$ & & 48 & & 10 & & 59 & & 43 \\
\hline $\mathrm{PHI}=1$ & 25 & $\%$ & & $\%$ & & $\%$ & & $\%$ \\
\hline
\end{tabular}

Figures in parentheses are standard errors

An interesting finding is shown by the smoking coefficient estimate. Contrary to what one would expect from adverse selection, smokers, whether single or in a family, are associated with significantly lower probabilities of having PHI in both years. One possible explanation is that this reflects the lower risk aversion of smokers which plausibly dominates the higher health care needs consideration.

The signs of the estimated coefficients for other important explanatory variables such as doctor visits and the number of chronic conditions are more consistent with the usual expectations that they capture expected future medical needs. All else equal, units with higher expected medical needs have on average higher probabilities for being covered with PHI. One particularly interesting finding from the family equation is that the coefficients on doctor visits suggest that, in 1995, the expected medical needs of the children seem to be more important than those of the adults. Also, in the same 
year, demand seems to increase with family size but at the same time decrease with the number of young children (aged less than six).

Some more interesting results can be further revealed from comparisons between the 1995 and 2001 estimated coefficients. For example, if the policy changes induce more individual from the lower risk group to join PHI membership, we should see that the importance of the risk related variables to be lower in 2001. The doctor visits variables of singles and families, both adult and children, seem to indicate that this is the case. Except for "drvisit3" and "drvisit4" of families, the magnitudes of the estimated coefficients decrease between the two periods. However, the other risk-related variables, e.g., number of chronic conditions and smoking, seem to provide contradicting evidence.

Other interesting results come from the comparison of the age coefficients. Since all other policy changes except LHC are basically age independent, we would expect that on average, the distance between LHC target group (age 30-65) and LHC non-target group (age 18-29 and age 65+) would increase. In other words, since the base age group (age 40-44) belongs in the LHC target group, the estimated coefficients of non-target group should be lower in 2001. The evidence seems to support this.

Based on the coefficients estimates, we estimate the increases in PHI membership which may result from the combination of policy initiatives introduced between 1995 and 2001. More specifically, we create a hypothetical situation by applying the 2001 NHS data on the 1995 coefficient estimates. In so doing, we in effect "force" singles and families in 2001 to apply the decision rules as embodied in the 1995 coefficients, which presumably would not reflect the effects of policy initiatives introduced after 1995. In this sense, we create a hypothetical or counterfactual scenario to which we can compare the actual predicted probabilities for 2001 and arrive at estimates of the effects of the policy initiatives introduced after 1995.

Table 3 presents the results of the counterfactual analysis. Looking at the last column of the table, $36.9 \%$ of single individuals had PHI in 2001, and this consists of $21.4 \%$ who would have had PHI in 2001 even if the government introduced no new policy initiatives, and the other $15.5 \%$ who took up PHI in response to the new policy initiatives. Similarly 
for families, $51.9 \%$ had PHI in 2001, and this can be decomposed into $21.8 \%$ who would have had PHI even if there were no new policy initiatives, and the remaining $30.1 \%$ who were new to PHI memberships, presumably in response to the new policy initiatives.

Table 3: Proportion of Population with PHI in 2001*

\begin{tabular}{llccc}
\hline & \multicolumn{5}{c}{ Age Group } \\
\cline { 2 - 5 } & $18-29^{* *}$ & $30-69$ & $70+$ & All \\
\hline Singles & 3.7 & 25.0 & 8.2 & 36.9 \\
Total & 0.8 & 15.6 & 4.9 & 21.4 \\
If no policy change & 2.9 & 9.4 & 3.2 & 15.5 \\
Attributed to new policies & & & &
\end{tabular}

\section{Families}

\begin{tabular}{lllll} 
Total & 3.7 & 44.0 & 4.1 & 51.9 \\
If policy change & 0.3 & 20.6 & 1.0 & 21.8 \\
Attributed to new policies & 3.4 & 23.5 & 3.2 & 30.1 \\
\hline
\end{tabular}

* all figures are percentage points

** for families: $20-29$

Columns 2-4 of Table 3 provide the breakdown of the above figures into three age groups: 18-29, 30-69, and 70+. For example, from columns 3 Table 3, out of the 15.5 percentage points of singles who took up PHI in response to the new policies shown in the last column of the same table, approximately 9.4 percentage points (or $61 \%$ of 15.5 ) were from Group 2, the LHC target group. It can be argued that other policies beside LHC might also contribute to this 9.4 percentage points of PHI enrollments in Group 2. However, in order to arrive at an estimate of an upper bound of the LHC effects, we will ignore this possibility. Thus, if we attribute all new PHI enrollees in the LHC target group to LHC, we can say that at most $61 \%$ of all singles who responded to the new policy initiatives were due to LHC. That is, $61 \%$ is an upper bound of the total effects of LHC on singles. By the same reasoning, we obtain the upper bound of the LHC effect for families as $78 \%$. Notice however that the true effects are probably much lower than 
these upper bounds since the latter are obtained on the assumption that individuals and families in Age Group 2 responded only to LHC and not other policy initiatives. ${ }^{3}$

Before we proceed to the estimation of the lower bounds, we note that the first and third groups defined above are not, by policy design, LHC target groups. Furthermore, note that the first group is probably the least risky group in terms of expected medical needs. On the other hand, the third group can be considered as the highest risk group. In addition, the first two groups' risk profiles might be more similar than the last two groups'. In other words, controlling for all other observable PHI determinants, the first group predicted probabilities would be a better reflection of the effects of non-LHC policies, chiefly the $30 \%$ premium rebate, than the last group's. Also, we can expect that the difference between Groups 1 and 2 is a much more accurate reflection of the LHC effects than the difference between Groups 2 and 3. Therefore, we will use the difference between the first two groups to arrive at the lower bound estimates.

We first need to isolate the effects of LHC from those PHI members attributed to the new policies by taking into account the marginal effects of the age variables. Table 4 presents the average marginal effects of all age variables with respect to the base age group (age 40-44) in 2001. In addition, the table also gives the predicted probabilities for the base age group so that the predicted probabilities of all other age groups can be inferred directly from their marginal effects. These age-dependent variations in predicted probabilities will allow the effects of LHC to be separated from other effects.

Looking at the within group averages on Table 4, the predicted probabilities of having PHI for for singles in groups 1 and 2 are $11.5 \%$ and $37.2 \%$, respectively. For families, the corresponding figures are $27.6 \%$ and $58.3 \%$. Then, by our previous reasoning, the difference between the average probabilities of Groups 1 and 2 can be treated as the part of predicted probabilities in Group 2 attributable to LHC. These differentials are 25.7 (=37.2 - 11.5) percentage points and 21.1 percentage points for singles and families, respectively. That is, expressed as a percentage of the within group average probabilities,

\footnotetext{
${ }^{3}$ An age-based difference-in-difference or a regression discontinuity analysis might be used to arrive at a less biased estimate of the LHC effect. However, the difficult part will be in defining the age group of families (see, for example, Palangkaraya and Young (2004).
} 
Table 4: Marginal Effects of Age on Probability of Having PHI in 2001

\begin{tabular}{|c|c|c|c|c|}
\hline \multirow[t]{3}{*}{ Variables } & \multicolumn{2}{|c|}{ Single } & \multicolumn{2}{|c|}{ Family } \\
\hline & Marginal & & Marginal & \\
\hline & effects & $\operatorname{Pr}[\mathrm{PHI}=1]$ & effects & $\operatorname{Pr}[\mathrm{PHI}=]$ \\
\hline \multicolumn{5}{|l|}{ Reference Group } \\
\hline Age40-44* & - & 0.2861 & - & 0.5099 \\
\hline \multicolumn{5}{|l|}{ Age Group 1} \\
\hline Age18-19 & -0.1960 & 0.0901 & - & - \\
\hline Age20-24 & -0.1555 & 0.1306 & -0.2762 & 0.2337 \\
\hline Age25-29 & -0.1610 & 0.1251 & -0.1914 & 0.3185 \\
\hline Within group average & & 0.1153 & & 0.2761 \\
\hline \multicolumn{5}{|l|}{ Age Group 2} \\
\hline Age30-34 & -0.0164 & 0.2697 & -0.0781 & 0.4317 \\
\hline Age35-39 & 0.0233 & 0.3094 & -0.0420 & 0.4679 \\
\hline Age45-49 & 0.0526 & 0.3387 & 0.0472 & 0.5571 \\
\hline Age50-54 & 0.0714 & 0.3576 & 0.0942 & 0.6041 \\
\hline Age55-59 & 0.1197 & 0.4059 & 0.1435 & 0.6534 \\
\hline Age60-64 & 0.1491 & 0.4352 & 0.1821 & 0.6919 \\
\hline Age65-69 & 0.2031 & 0.4892 & 0.2408 & 0.7506 \\
\hline Within group average* & & 0.3722 & & 0.5833 \\
\hline \multicolumn{5}{|l|}{ Age Group 3} \\
\hline Age70-74 & 0.1941 & 0.4803 & 0.2616 & 0.7715 \\
\hline Age75-79 & 0.1552 & 0.4413 & 0.1610 & 0.6709 \\
\hline Age80 \& above & 0.1864 & 0.4726 & 0.1805 & 0.6904 \\
\hline
\end{tabular}

* inclusive of reference age group 
LHC accounts for approximately $68 \%$ and $53 \%$ of the average probabilities of having PHI for singles and families in Group 2.

We use the above probabilities to break down the proportion of population in the LHC target group as shown in the third column of Table 5. The first row under each Singles or Families heading is nothing but the proportion of new PHI members presented in Table 3 earlier. In particular, the value in the last row under each heading in column 3 of Table 5 represents a lower bound estimate of the proportion of population new to PHI due to LHC. For example, for singles, $6.4(68 \% \times 9.4)$ percentage points is the estimated effects of LHC. This is approximately $41 \%$ of all 15.5 percentage points singles who responded to the new policy initiatives regardless. It is quite possible that, for some reasons, the effects of LHC spillover into the non-targeted age groups. Since the previous figure is obtained by assuming that LHC does not affect the other two age groups, then it is more appropriate to be treated as the lower bound of the LHC effect on singles. By the same reasoning, we arrive at the lower bound for families, which is estimated to be around $42 \%(=11.0 / 23.5)$. We need to stress that the true magnitude of the total effects would be closer to the lower bound than to the upper bound. This is because the potential spillover effects of LHC on Groups 1 and 3, say because of confusion in understanding the regulations, are most likely to be smaller than the potential effects of other non-LHC related, age-correlated determinants that we ignored in estimating the upper bounds.

Lastly, it is worth noting that a weighted average scheme can be used to obtain an interval estimate for the total effect of LHC on the whole population. Since approximately $20 \%$ of the respondents in the 2001 NHS are singles, thus a 1:4 ratio seems appropriate. Using this weight, we estimate that LHC accounts for between $42 \%$ and $75 \%$ of the rise in PHI membership in the Australian population. For the same reasons we mentioned above, we think the true value is likely to be much closer to the former than the latter. 
Table 5: Proportion of Population "New" to PHI in 2001*

\begin{tabular}{llrrr} 
& \multicolumn{5}{c}{ Age Group } \\
\cline { 2 - 5 } & $18-29^{* *}$ & $30-69$ & $70+$ & All \\
\hline Singles & & & & \\
Proportion with PHI in 2001, but " not" in 1995 & 2.9 & 9.4 & 3.2 & 15.5 \\
$\quad$ Attributed to the 30\% Premium Rebate & 2.9 & 3.0 & 3.2 & 9.1 \\
$\quad$ Attributed to the Lifetime Health Cover & 0 & 6.4 & 0 & 6.4 \\
& & & & \\
Families & & & & \\
Proportion with PHI in 2001, but " not" in 1995 & 3.4 & 23.5 & 3.2 & 30.1 \\
$\quad$ Attributed to the 30\% Premium Rebate & 3.4 & 11.0 & 3.2 & 17.6 \\
$\quad$ Attributed to the Lifetime Health Cover & 0 & 12.5 & 0 & 12.5 \\
\hline
\end{tabular}

* all figures are percentage points

** for families: $20-29$

\section{Conclusion}

Economic efficiency alone dictates that if a policy costs less and yields higher desired effects, then it should be a preferred option. Among the recent Australian private health insurance policy initiatives, the Lifetime Health Cover policy costs almost nothing while the $30 \%$ premium rebate costs approximately $\$ 2$ billions per year. The crucial question is whether or not the latter, a much more expensive policy, is completely ineffective, as claimed by many authors and policy commentators.

By looking at time series data and noting the date of implementation, one gets the impression that Lifetime Health Cover seems to account for most of the increase in private health insurance take-up rates. Such a conclusion could be warranted if not for the following considerations. First, the $30 \%$ premium rebate pre-dated Lifetime Health Cover, thus the jump in private health insurance memberships one observes may very well be due to the rebate as well. Second, Lifetime Health Cover is very specific in its target groups and these target groups account for less than $72 \%$ of private health insurance 
membership in 2001. Moreover, a significant proportion of those in the target groups were in fact already covered by private health insurance before those policy initiatives were implemented. Thus, the increase in private health insurance memberships in these groups may not account for the bulk of the overall increase in private health insurance memberships. Third, data from the 2001 National Health Survey indicate that only a small fraction of people in the target groups cited Lifetime Health Cover as their reasons for purchasing private health insurance.

Mindful of these arguments, we proceed to untangle the effects of the Lifetime Health Cover from other policy initiatives. We do so by estimating private health insurance demand models using micro-level data before and after the policy initiatives were in place. The main findings are that Lifetime Health Cover accounts for at least $42 \%$ and at most $75 \%$ of the overall increase in private health insurance membership. We also argue that the true share of Lifetime Health Cover would probably be much closer to the lower bound. Thus, we can conclude that the contribution of the $30 \%$ premium rebate could be far more substantial than most authors and commentators believe.

There are several possibilities of how this study can be improved. First, in modeling private health insurance demand, we do not consider the fact that PHI in Australia is secondary to a freely available alternative, the Medicare. A more careful modeling of such duplicate cover, for example see Vera-Hernandez (1999), may result in more precise estimates. Another important improvement can be made by carefully taking into account the effects of the $1 \%$ Medicare levy on high income earners. This can be done, for example, by using the regression discontinuity approach by exploiting the fact that the levy only kicks in after a specific income threshold. This should result in a more precise accounting of the contributions of each policy initiative. 


\section{References}

[1] Barrett, G. F. and R. Conlon (2003), "Adverse selection and the decline in private health insurance coverage in Australia: 1989-95," The Economic Record, 79 (246), 279-296.

[2] Butler, J. R. G. (2002), "Policy change and private health insurance: did the cheapest policy do the trick," Australian Health Review, 25 (6), 33-41.

[3] Cameron, A. C. and P. K. Trivedi (1991), "The role of income and health risk in the choice of health insurance: Evidence from Australia," Journal of Public Economics, $45,1-28$.

[4] Ducket, S. J. and T. J. Jackson (2000), "The new health insurance rebate: an inefficient way of assisting public hospitals," Medical Journal of Australia, 172 (9), 439-442.

[5] Frech, H. E. III, S. Hopkins, and G. Macdonald (2003), "The Australian private health insurance boom: was it subsidies or liberalised regulation?" Economic Papers, $22(1), 58-64$.

[6] Hopkins, S. and M. P. Kidd (1996), "The determinants of the demand for private health insurance under Medicare," Applied Economics, 28, 1623-1632.

[7] Industry Commission (1997), Private Health Insurance, PC Report No. 57, (http://www.pc.gov.au/ic/inquiry/57privatehealth/finalreport/57privatehealth.pdf).

[8] Palangkaraya, A. and J. Yong (2004), "How effective is 'Lifetime Health Cover' in raising private health insurance coverage in Australia? A regression discontinuity approach," Working Paper, Melbourne Institute of Applied Economic \& Social Research, University of Melbourne.

[9] Vera-Hernandez, A. M. (1999), "Duplicate coverage and demand for health care: The case of Catalonia," Health Economics, 8, 579-598. 